\title{
Oestrogen plus progestogen increased risk of breast cancer in postmenopausal women
}

Chlebowski RT, Hendrix SL, Langer RD, et al. Influence of estrogen plus progestin on breast cancer and mammography in healthy postmenopausal women: the Women's Health Initiative Randomized Trial.JAMA 2003;289:3243-53.

\section{QUESTION: In postmenopausal women, does oestrogen plus progestogen hormone therapy $(\mathrm{HT})$ increase the risk of abnormal mammographic results and diagnoses of breast cancer?}

\section{Design}

Randomised (allocation concealed*), blinded (clinicians, participants, data collectors, outcome assessors, and monitoring committee),* placebo controlled trial with a mean 5.6 year follow up (Women's Health Initiative [WHI]).

\section{Setting}

40 US clinical centres.

\section{Participants}

16608 postmenopausal women who were $50-79$ years of age (mean age 63.3 y). Exclusion criteria were previous hysterectomy, breast cancer, or probable survival $<3$ years. Follow up data were available for 15931 women $(95.9 \%)$.

\section{Intervention}

Women were allocated to 1 daily tablet of conjugated equine oestrogen, $0.625 \mathrm{mg}$, and medroxyprogesterone acetate, $2.5 \mathrm{mg}(\mathrm{n}=8506)$, or placebo $(\mathrm{n}=8102)$.

\section{Main outcome measures}

Incidence of breast cancer (total, invasive, and in situ) and abnormal mammogram results.

Sources of funding: National Heart, Lung and Blood Institute and Wyeth-Ayerst

Research Laboratories.

For correspondence: Dr R T Chlebowsi Harbor-UCLA

Research and

Education Institute

Torrance, CA, USA.

rchlebowski@rei.edu

Abstract and

commentary also

appear in ACP Journal Club.

\section{Main results}

Analysis was by intention to treat. Women who received HT had a greater incidence of total and invasive breast cancer than did women who received placebo; in situ breast cancer cases were not increased (table). The increase in invasive breast cancer with HT was seen across almost all risk categories. Invasive breast tumours were larger in the HT group (mean $1.7 \mathrm{~cm} v 1.5 \mathrm{~cm}$, $\mathrm{p}=0.04)$ and were diagnosed at a more advanced stage (regional or metastatic, compared with local, 25\% v 16\%, $\mathrm{p}=0.04)$ than in the placebo group. Women who received HT also had a higher proportion of abnormal mammogram results. The difference was seen at 1 year $(9.4 \% v 5.4 \%, \mathrm{p}<0.001)$ and continued throughout the study (total study period $31.5 \%$ v 21.2\%, $\mathrm{p}<0.001$ ).

Oestrogen plus progestogen hormone therapy (HT) v placebo for incidence of breast cancer in postmenopausal women at mean 5.6 yearst

\begin{tabular}{lccc} 
Outcomes & \multicolumn{2}{c}{ Cases of breast cancer } & Hazard ratio $(95 \% \mathrm{CI})$ \\
\hline & HT & Placebo & \\
\hline All breast cancer & 245 & 185 & $1.24(1.02$ to 1.50$)$ \\
\hline Invasive breast cancer & 199 & 150 & $1.24(1.01$ to 1.54$)$ \\
\hline In situ breast cancer & 47 & 37 & $1.18(0.77$ to 1.82$)$ \\
\hline
\end{tabular}

\section{Conclusion}

In postmenopausal women, oestrogen plus progestogen hormone therapy increased cases of total and invasive breast cancer and abnormal mammogram results.

*See glossary.

\section{COMMENTARY}

The study by Chlebowski et al, gives detailed information regarding breast cancer risk in late postmenopausal women using oestrogen plus progestogen HT. No increased risk of breast cancer death was reported in HT users, and the diagnosis of metastatic breast cancer was observed in $1 \%$ of users compared with $2 \%$ of placebo users. The authors emphasised that HT users had larger tumours than placebo users $(0.2 \mathrm{~cm}$ larger with a $10 \%$ greater node involvement $[\mathrm{p} \leq 0.04])$. The recently released Million Women Study ${ }^{1}$ found that current HT therapy, particularly oestrogen plus progestogen, was associated with increased risk of breast cancer. However, past use was not associated with such risk. It is becoming clear that current oestrogen plus progestogen HT carries greater breast cancer risk than oestrogen alone. Of note, the oestrogen only arm of the WHI is still ongoing.

Women and clinicians are receiving the message that HT increases breast cancer. Placing this in perspective for the menopausal woman ${ }^{2}$ is important because there will continue to be women who want to use HT. In women with a uterus requiring progestogen opposition, it is likely that less use of synthetic progestogens and more use of localised vaginal uterine preparations will occur, particularly if it is proven that oestrogen alone is safer from the breast cancer perspective.

Because HT users had a substantially greater risk of abnormal mammography results than placebo users $(9.4 \% \mathrm{v}$ $5.4 \%, \mathrm{p}<0.001$ ), should women on HT stop using it for 2 weeks before an annual mammogram? It has been suggested that short term cessation of HT improves mammographic specificity. ${ }^{3}$ Although screening mammography is the current gold standard, a way to detect breast cancer before finding an abnormality on mammography is needed. Ductal lavage, recently approved by the US Food and Drug Administration, can be used as a risk assessment tool for women at high risk of breast cancer who have negative results on mammography. This may help menopausal women to make well informed decisions regarding HT, the use of tamoxifen for breast cancer chemoprevention, and other decision options related to the breast ${ }^{4}$

Holly L Thacker, MD

Cleveland Clinic Foundation, Cleveland, Ohio, USA

1 Beral V. Breast cancer and hormone-replacement therapy in the Million Women Study. Lancet 2003;362:419-27.

Batur P, Thacker HL, Moore HC. Discussing breast cancer and hormone replacement therapy with women. Cleve Clin J
Med 2002:69:838, 840,843-4 passim.

3 Harvey JA, Pinkerton JV, Herman CR. Short-term cessation of hormone replacement therapy and improvement of mammographic specificity. J Natl Cancer Inst 1997;89:16235 .

4 O'Shaughnessy JA, Ljung BM, Dooley WC, et al. Ductal lavage and the clinical management of women at high risk for breast carcinoma: a commentary. Cancer 2002;94:292-8. 